\title{
Narrative review of gene modification: applications in three-dimensional (3D) bioprinting
}

\author{
Bowen Fu ${ }^{1,2 \#}$, Jianlin Shen ${ }^{1,3 \#}$, Yu Chen ${ }^{4 \#}$, Yanjiao Wu ${ }^{5}$, Heshi Zhang ${ }^{6}$, Huan Liu ${ }^{7}$, Wenhua Huang ${ }^{1,2}$ \\ ${ }^{1}$ Department of Orthopedics, Center for Orthopaedic Surgery, The Third Affiliated Hospital of Southern Medical University, Guangzhou, China; \\ ${ }^{2}$ School of Basic Medical Sciences, Southern Medical University, Guangdong Provincial Key Laboratory of Medical Biomechanics, Guangdong \\ Provincial Medical 3D Printing Application Transformation Engineering Technology Research Center, Guangzhou, China; ${ }^{3}$ Department of \\ Orthopaedics, Affiliated Hospital, Putian University, Putian, China; ${ }^{4}$ Central Laboratory, Affiliated Hospital of Putian University, Putian, China; \\ ${ }^{5}$ Department of Orthopedics, Shunde Hospital of Southern Medical University Guangzhou, China; ${ }^{6}$ Department of Vessel \& Breast \& Thyroid \\ Surgery, Hospital (TCM) Affiliated to Southwest Medical University, Luzhou, China; ${ }^{7}$ National Traditional Chinese Medicine Clinical Research \\ Base, The Affiliated Traditional Chinese Medicine Hospital of Southwest Medical University, Luzhou, China \\ Contributions: (I) Conception and design: B Fu, J Shen, Y Chen; (II) Administrative support: H Liu, W Huang; (III) Provision of study materials or \\ patients: B Fu, H Liu, Y Wu, Y Chen; (IV) Collection and assembly of data: B Fu, J Shen;(V) Data analysis and interpretation: B Fu, J Shen, H Liu, \\ Y Wu, Y Chen; (VI) Manuscript writing: All authors; (VII) Final approval of manuscript: All authors. \\ \#These authors contributed equally to this work. \\ Correspondence to: Wenhua Huang. School of Basic Medical Sciences, Southern Medical University, Guangdong Provincial Key Laboratory of Medical \\ Biomechanics, Guangdong Provincial Medical 3D Printing Application Transformation Engineering Technology Research Center, Guangzhou \\ 510515, China. Email: huangwenhua2009@139.com; Huan Liu. National Traditional Chinese Medicine Clinical Research Base, The Affiliated \\ Traditional Chinese Medicine Hospital of Southwest Medical University, Luzhou 646000, China. Email: 20016040@163.com.
}

Objective: This article focused on the application scenarios of three-dimensional (3D) bioprinting and gene-editing technology in various medical fields, including gene therapy, tissue engineering, tumor microenvironment simulation, tumor model construction, cancer regulation and expression, osteogenesis, and skin and vascular regeneration, and summarizing its development prospects and shortcomings.

Background: 3D bioprinting is a process based on additive manufacturing that uses biological materials as the microenvironment living cells. The scaffolds and carriers manufactured by 3D bioprinting technology provide a safe, efficient, and economical platform for genes, cells, and biomolecules. Gene modification refers to replacing, splicing, silencing, editing, controlling or inactivating genes and delivering new genes. The combination of this technology that changes cell function or cell fate or corrects endogenous mutations and 3D bioprinting technology has been widely used in various medical field.

Methods: We conducted a literature search for papers published up to March 2021 on the gene modification combined with 3D bioprinting in various medical fields via PubMed, Web of Science, China National Knowledge Infrastructure (CNKI). The following medical subject heading terms were included for a MEDLINE search: "3D printing/gene editing”, "3D printing/genetic modification", "3D printing/seed cell”, "bioprinting/gene editing", "bioprinting/genetic modification", "bioprinting/seed cell”, "scaffold/gene editing", "scaffold/genetic modification", "scaffold/seed cell”, "gene/scaffold”, "gene/bioprinting”, "gene/3D printing". Quantitative and qualitative data was extracted through interpretation of each article.

Conclusions: We have reviewed the application scenarios of 3D bioprinting and gene-editing technology in various medical fields, it provides an efficient and accurate delivery system for personalized tumor therapy, enhancing the targeting effect while maintaining the integrity of the fabricated structure. It exhibits significant application potential in developing tumor drugs. In addition, scaffolds obtained via 3D bioprinting provide gene therapy applications for skin and bone healing and repair and inducing stem cell differentiation. It also considers the future development direction in this field, such as the emergence and development of

$\wedge$ ORCID: 0000-0002-0965-2799. 
gene printing, 4D printing. The combination of nanotechnology and gene printing may provide a new way for future disease research and treatment.

Keywords: Three-dimensional bioprinting (3D bioprinting); genetic modification; scaffold

Submitted Jun 03, 2021. Accepted for publication Aug 23, 2021.

doi: $10.21037 / \mathrm{atm}-21-2854$

View this article at: https://dx.doi.org/10.21037/atm-21-2854

\section{Introduction}

Three-dimensional (3D) printing technology, also known as rapid prototyping technology, is used to print metal powder, photosensitive resin, and other bondable materials layerby-layer according to computer data, finally superimposing them into a $3 \mathrm{D}$ entity. The $3 \mathrm{D}$ printing process mainly includes $3 \mathrm{D}$ printing technology, selective laser sintering technology, selective laser melting technology, and fused deposition modeling technology. These techniques present advantages, such as individualization, precision, and remote operability, which are particularly suitable for application in the medical field (1). Since the 1980s, the rapid development of $3 \mathrm{D}$ printing has been hailed as "one of the important symbols of the third industrial revolution".

$3 \mathrm{D}$ bioprinting is a branch of $3 \mathrm{D}$ printing and has prompted renewed research interest. Compared with $3 \mathrm{D}$ printing, the main difference lies in the type of material. Traditional 3D printing technology employs materials, such as polymers, metals, alloys, plastics, ceramics, and resins, while bioprinters use biologically active substances, biological materials, or bionic molecules. As an extension of $3 \mathrm{D}$ printing, 3D bioprinting aims to print bionic tissues or cell models that can simulate the functions and structures of target tissues, such as skin tissue, blood vessels, and multicellular structures (2). They also contain liquid, paste, or gel-type scaffolds as a matrix or colloid for cell growth (e.g., cell hydrogel and cell suspension) (3). The main materials currently used for bioprinting are collagen, fibrin, silk, chitosan, alginate, gelatin, hyaluronic acid, and methacrylic acid gelatin (4). The primary manufacturing technologies include inkjet, laser, bioplotting, and fuseddeposition modeling (FDM).

Of these, inkjet-based bioprinting (also known as dropon-demand printing, drop-by-drop, or drop-on-demand bioprinting) is one of the oldest printing methods that still show significant promise in $3 \mathrm{D}$ printing (5). Inkjet printing is a non-contact copy strategy based on the deposition of biological ink droplets. The manufacturing strategy for producing ink droplets relies on three different methods. These include piezoelectric inkjet (acoustic), thermal inkjet, and electrostatic bioprinting (6). In addition to nonbiological materials, the encapsulated cell droplets can also be printed layer-by-layer and assembled into structures (7). Piezoelectric bioprinters use piezoelectric actuators that eject liquid droplets through printer nozzles to generate sound waves in the bio-ink chamber (8). Thermal inkjet bioprinters consist of a fluid chamber and one or more nozzles, while heat and pressure pulses are generated in the bio-ink chamber. The pressure causes picoliter droplets to be ejected from the nozzle orifice (9). In electrostatic bioprinters, the droplets are generated by voltage pulses between the platen and the electrodes (5). Inkjet-based bioprinting is fast, inexpensive, exhibits high cell viability, and is compatible with non-biological and biological materials (10). However, some limitations exist, such as the use of high-viscosity materials that may cause nozzle clogging, resulting in unnecessary pressure problems (11). In addition, since this method typically uses low-viscosity bio-inks, the mechanical strength of the printed structure is usually inferior to that of the target tissue (6).

The freeform reversible embedding of suspended hydrogels (FRESH) represents a newly emerging 3D printing technology. It uses a thermoreversible support bath to deposit hydrogels in complex 3D biological structures and employs open-source tools to render it a highly adaptable and cost-effective biological additive manufacturing (AM) platform. The key innovation of FRESH is depositing and embedding the hydrogel being printed into a second hydrogel support bath that maintains the desired structure during the printing process, significantly improving the printing fidelity. The support bath is composed of gelatin particles, acting as Bingham plastics during the printing process. They behave as rigid bodies under low shear stress and viscous fluids under higher shear stress. Consequently, when the needle-shaped nozzle passes through the bathtub, there is almost no mechanical resistance, but the hydrogel 
extruded from the nozzle and deposited in the bathtub is fixed in place. Therefore, soft materials that collapse when printed in the air can easily be kept in the expected $3 \mathrm{D}$ geometry. This process is performed in a sterile, watercontaining buffer environment compatible with the cells, showing that the cells can be squeezed from the printer nozzle through the hydrogel and remain viable. Once the entire $3 \mathrm{D}$ structure is freshly printed, the temperature rises to $37^{\circ} \mathrm{C}$, which is cell-friendly, allowing the gelatin support bath to melt in a non-destructive manner. FRESH enables the direct 3D printing of biologically relevant hydrogel inks, including alginate, fibrin, collagen type I, and Matrigel within a fugitive support bath (12).

Genetically modified cells are currently studied as a new type of 3D bioprinting material. Genetic modification refers to replacing, splicing, silencing, editing, controlling, or inactivating genes and delivering new genes. This technique of changing cell function or cell fate or correcting endogenous mutations has been widely used in various fields of medicine (13). Using RNA interference (RNAi) for gene silencing and the introduction of other nucleic acids (such as siRNA, miRNA, or shRNA) unlocks targeted treatments for various diseases (such as viral infectious diseases, neuroblastoma, and ophthalmological diseases) (14). Transgenic stem cells are used as therapeutic agents or gene delivery systems to change the cell activities, mechanisms, and molecular structure of wounds or target tissues during wound treatment, skin regeneration, or anti-scarring therapy (15). However, gene therapy is a complex medical field, facing many limitations in terms of delivering genes to target sites and subsequent treatment. For example, viral vectors can produce toxicity or immune responses in the body. Similarly, non-viral vectors may exhibit low transfection problems, while the two vectors used for delivery may exhibit a lack of purity, off-target effects, a lack of efficiency, and possess limited DNA carrying capacity $(14,16)$.

$3 \mathrm{D}$ bioprinting provides a real-time, diverse, and efficient platform for gene modification technology. It can produce scaffolds containing growth factors, stem cells, and nucleic acids, providing a stable environment releasing these molecules in the body $(17,18)$. Furthermore, the modified gene expression or enhancement achieved by $3 \mathrm{D}$ scaffolds also provide new techniques for skin regeneration, bone tissue engineering, and wound healing $(15,19)$. This article aims to review the application of gene modification combined with $3 \mathrm{D}$ bioprinting in various medical fields while summarizing its development prospects and shortcomings. We present the following article in accordance with the Narrative Review reporting checklist (available at https://dx.doi.org/10.21037/atm-21-2854).

\section{Methods}

We conducted a literature search for papers published up to March 2021 on the gene modification combined with 3D bioprinting in various medical fields via PubMed, Web of Science, China National Knowledge Infrastructure (CNKI). The following medical subject heading terms were included for a MEDLINE search: "3D printing/gene editing", "3D printing/genetic modification", "3D printing/ seed cell”, "bioprinting/gene editing", "bioprinting/ genetic modification", "bioprinting/seed cell", "scaffold/ gene editing", "scaffold/genetic modification", "scaffold/ seed cell", "gene/scaffold", "gene/bioprinting", "gene/3D printing". Quantitative and qualitative data was extracted through interpretation of each article.

\section{Discussion}

\section{The application of gene modification based on $3 D$ bioprinting}

$3 \mathrm{D}$ bioprinting is currently widely applied in the fields of tissue or bone regeneration $(20,21)$, neuroblastoma cell culture systems (22), neural catheters or implant engineering (23), vaccine delivery (24), molecular diagnosis (25), surgical models (26), and other fields. In addition, 3D bioprinting technology exhibits significant potential for delivering genes to defective cells in tissue engineering, regenerative medicine, and treating various diseases, especially bone defects (27). The polymer materials used for 3D bioprinting are divided into two categories, namely non-biodegradable polymers and biodegradable polymers. Biodegradable polymers are used for tissue growth. Once the task is completed, the biomedical parts of the body are no longer needed. They degrade in the human body, for example, the ester bond of polyester is hydrolyzed. However, non-biodegradable polymers are used as structural implants. For example, some hard synthetic biodegradable polymers include poly(E-caprolactone) (PCL), polydioxanone (PDO) cyclic ketones, and polylactic acid (PLA) (28).

Similarly, the accelerated development of nanotechnology and gene-editing technology has allowed the creation of a new gene delivery system that can effectively transfect genes into host cells. Nanocarriers with advanced release characteristics help to efficiently transfer genes in various 


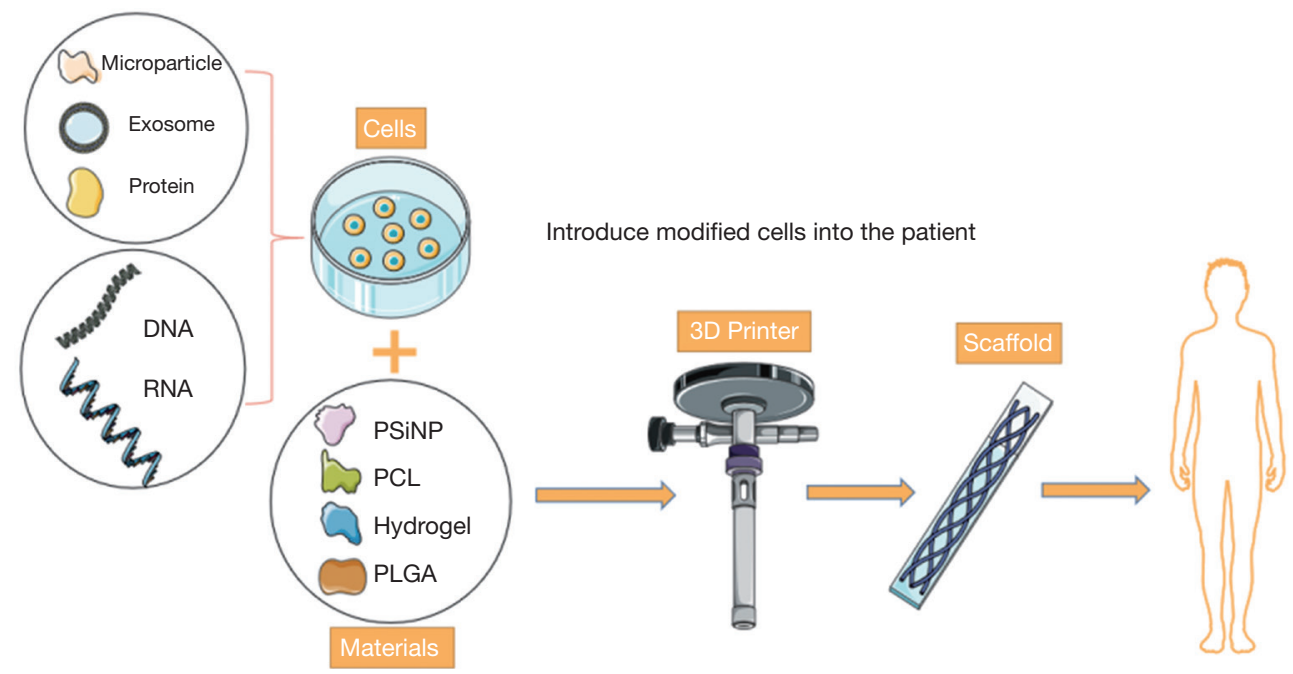

Figure 1 Preparation methods of gene editing and gene therapy scaffolds. PCL, poly(E-caprolactone); PLGA, polylactic acid-glycolic acid; PSiNP, porous silicon nanoparticles.

editing technologies (29). Combining nanocarriers with the scaffolds obtained via $3 \mathrm{D}$ bioprinting produces unique characteristics, allowing the activation of the gene, related molecule, and controlled release properties. The preparation methods for gene-editing and gene therapy scaffolds are summarized in Figure 1. The research progress regarding $3 \mathrm{D}$ bioprinting combined with gene-editing technology in the primary medical fields is discussed below.

\section{Application in oncology}

Bioprinting technology combined with genetic modification exhibit significant potential for tumor drug delivery, tumor treatment research, and tumor model construction in oncology. Furthermore, nanocarriers display advanced release characteristics. Combining the scaffolds obtained via $3 \mathrm{D}$ bioprinting with nanocarriers can be used to deliver related molecules and genes and regulate the release characteristics. Although camptothecin (CPT) is a highly effective chemotherapeutic compound that exhibits cytotoxic activity against several types of cancer cells $(30,31)$, it is non-specific and displays poor water solubility. It reached phase I and phase II clinical trials but failed due to severe side effects. A recent study loaded CPT into porous silicon nanoparticles (PSiNP) with the epidermal growth factor receptor (EGFR) targeting antibody (Ab), cetuximab, to produce a targeted, soluble nanoparticle-grade cancer treatment vector. Furthermore, this drug delivery system significantly reduced the transfer load of humanized bone and other organs (such as the lungs and liver) related to human metastatic breast cancer (32).

Tissue engineering methods combining genetic modification and bioprinting are equally effective in studying cancer-bone interactions in specific animal species. In 2017, Moreau et al. first studied the effect of tissueengineered bone (TEB) as a site for breast cancer cell metastasis, focusing on NOD/SCID mice (33). However, after transplantation in mice, humanized tissues were often substituted with endogenous mouse stromal cells (34). They did not consider the complexity of the bone tissue microenvironment, and the hematopoietic stem cell (HSC) transplantation was often accompanied by limited $\mathrm{T}$ cell reconstitution, especially when using cord blood HSCs (35). A recent study made progress regarding two aspects: (I) human preosteoblasts (HOBS) were planted on a calcium phosphate-coated medical-grade PCL stent to customize the intraosseous features in a highly controlled manner. Moreover, human bone marrow mesenchymal stem cells (BM-MSCs) and umbilical vein endothelial cells (HUVECs) were mixed with a gelatin methacryloyl (GelMA) hydrogel to form blood vessels in the bone tissue in the center of the tubular scaffold to develop a humanized model with a bone tissue microenvironment. (II) An engineered human breast cancer cell line that expressed human interleukin-7 (IL-7), interleukin-15 (IL-15), and granulocyte-macrophage colony-stimulating factor (GM-CSF) in vivo was used to overcome the problem of a lack of human cytokines after HSC transplantation (36). This kind of research using the tissue engineering microenvironment and genetically 
modified HSCs will help resolve the specific role of signal molecules during cancer metastasis in vivo and the regulation of the hematopoietic ecology.

In another study, a similar method has been used to simulate the tumor microenvironment (TME). Lactic acid is a glucose metabolism intermediate and is found to be a conjugate base, lactate under physiological $\mathrm{pH}$ is either a byproduct or an energy source for cancer cells, depending on the availability of oxygen (37). Therefore, it is necessary to design a TME simulation system that can replicate a key TME component, the endogenous lactic acid level, to understand the direct impact of the local lactic acid concentration on immune cells. Consequently, a peptide hydrogel loaded with GM-CSF and a degradable lactic acid-based polymer is used. The hydrogel environment encapsulates the polymer in a hydrated peptide network, hydrolyzing it into monomeric lactate and subsequently allowing the lactate to accumulate in the hydrogel. In addition, GM-CSF recruits innate immune cells, mainly dendritic cells and macrophages, into the hydrogel, blocking antigen-specific immunity against tumors. The results indicated that lactate accumulation in the hydrogel was comparable to the concentration observed in different tumor types. Furthermore, the infiltrating immune cells in the hydrogel loaded with lactide-based polysulfonic acid mucopolysaccharide showed a high degree of immunosuppression at 7 days (38). In summary, this TMEmimicking hydrogel system exhibits the potential for further exploring immune response based on systems biology. It is also possible to integrate immunotherapeutic drugs into this platform for discovery and mechanism research.

Thus far, research involving osteosarcoma (OS) in the traditional preclinical syngeneic and allogeneic operating system models has failed to promote the transformation of new therapeutic drugs into clinical practice. The speciesspecific incompatibility between human and murine organisms has become a concern (39). The differences in the nucleic acid and amino acid sequences of regulatory genes and proteins lead to crosstalk changes in intercellular and intracellular signaling networks, denoting the main reason for the unsuccessful translation of experimental research data (40). A recent study tissue-engineered a new orthotopic humanized OS model for the first time. The primary method involved planting human osteoblasts on medicalgrade polycaprolactone stents. These were implanted into polyethylene glycol-based hydrogels containing HUVECs, after which the in situ human origin was determined via $3 \mathrm{D}$ printed orthotopic humanized Tissue Engineering Bone
Construct (OhTEBC). The constructs were implanted into the femurs of NOD-SCID and NSG mice, while human CD34 cells were transplanted into the bone marrow of NSG mice. The growth of human OS was induced in the ohTEBC via direct injection of Luc-SAOS-2 cells (41). After the femurs were collected, micro-computed tomography and immunohistochemical staining revealed an organ with all the characteristics of human bones. Human-specific type I collagen (hs Col-I) staining showed the production of a human extracellular bone matrix. The presence of nuclei led to a positive staining result for human nuclear mitotic apparatus protein 1 (Hs NUMA), confirming that the bone cells in the bone matrix were of human origin. Flow cytometry confirmed the presence of human hematopoietic cells. In addition, the tumor marker expression was similar to that of human patients, while the recently discovered musculoskeletal gene, C12orf29, was expressed in the most common OS patient sample subtypes (41). This in situ bone tumor research platform can test patient-specific drug regimens before patient treatment and conduct controlled, predictive marker studies of primary bone tumors.

Although primary tumors and metastatic tumors secondary to bone tissue are removed during surgery, the remaining tumor tissue may cause tumor recurrence (42). Since a stent usually covers the surgical space to provide mechanical support, the release of chemotherapeutic drugs through this type of stent to kill the remaining cancer cells becomes essential to the treatment (43). A previous study developed a 3D bioprinting PCL scaffold composed of doxorubicin and chitosan/siRNA nanoparticles. The siRNA was used to induce gene silencing in tumor cells to prevent tumor resistance while inducing a synergistic effect with adriamycin on cancer cell death. The results showed that the scaffold displayed explosive release in glioma (U251) cells and non-small cell lung cancer (H1299), followed by gradual siRNA release, inducing sequence-specific gene silencing. When chitosan nanoparticles were combined with doxorubicin, a synergistic decrease in cancer cell viability was observed. It is speculated that this synergy can be attributed to the increased uptake of doxorubicin, cell membrane destabilization, and the combined use of drugs to induce apoptosis or necrosis (44).

\section{Application in orthopedics}

Traditional orthopedic surgery has a poor therapeutic effect on rotator cuff tendon-bone injuries. The rotator cuff tendon fails to heal after repair in more than $90 \%$ of patients $(45,46)$. Studies have shown that the 
differentiation ability of BM-MSCs can improve the rotator cuff functionality after tear repairs. In addition, bone morphogenetic protein 12 (BMP-12), also known as growth differentiation factor 7 , it has a substantial impact on tendon repair and tendon-like tissue formation $(47,48)$. A recent study developed a $3 \mathrm{D}$ printed polylactic acidglycolic acid (PLGA) scaffold, on which they planted rabbit BM-MSCs with high BMP-12 expression. Compared with the control group, the BM-MSCs transfected with the BMP-12 overexpression vector significantly increased the expression of biomarkers related to tendon differentiation. The collagen fibers, chondrocytes, and fibrocartilage at the junction of the tendon and bone increased significantly, compared with the control group. Moreover, the animals in the experimental group displayed better biomechanical effects at 4, 8, and 12 weeks after surgery (49).

Another study involved implants for personalized cell therapy for bone repair, loaded with the transcription factor, GET-RUNX2, polymer (polyethylene glycol/polyethylene glycol) microparticles, and temperature-sensitive materials, which were mixed and then co-printed on mesenchymal stem cells. In vitro studies have shown that the sustained release of RUNX2 from the encapsulated microparticles induces a higher osteogenic effect in seed stem cells under the action of transcription factors. A scaffold was used during in vivo experiments to fill the defect of the distal femur of nude mice, significantly increasing the volume of high-density bone formation after 6 weeks (50).

The research strategy mentioned above mainly combines scaffolds, growth factors, and seed cells to regulate bone formation. However, the clinical application of cell-based tissue engineering faces many challenges, such as activity and cell sources, immune rejection, extended treatment time, and high cost (51). A recent study wrapped the vascular endothelial growth factor $(V E G F)$ gene in ATDC5derived exosomes to construct engineered exosomes that were gene-activated. The specific extracellular anchor peptide, cp05, was used as a flexible linker to effectively combine the engineered extracellular nanoparticles with the porous bone scaffold obtained via 3D bioprinting. The results showed that a significant amount of new bone tissue and many blood vessels were evident in the bone defect of the rat 12 weeks after implanting the engineered exosome scaffold. Contrarily, the control group scaffold was mainly filled with soft, fibrous connective tissue containing randomly oriented low-density collagen fibers and blood vessels. Therefore, the engineered exosomes could be used as an osteogenic matrix to induce the differentiation of mesenchymal stem cells in an osteogenic direction and as a gene carrier for the controlled release of the $V E G F$ gene to rebuild the vascular system (52).

\section{Application in dermatology}

The skin surface plays a crucial role in maintaining body fluid balance and regulating body temperature. It provides a barrier against the outside world (especially against pathogenic microorganisms) and regulates many metabolic processes (53). Burns represents the leading cause of skin damage. According to the latest report of the World Health Organization, about 265,000 people die from thermal burns every year (54). Various innovative treatment methods, such as stem cells and other types of cell therapies, as well as gene-editing combined with $3 \mathrm{D}$ scaffold therapies, have significantly improved wound healing. A previous study combined MSCs with the hepatocyte growth factor (HGF), a chemical inducer of MSC loaded in collagen and silk fibroin scaffolds, to explore the suitability of MSCs for treating chronic wounds or burns. Here, the active HGF was released from the scaffold, inducing direct cell migration and leading to the endogenous recruitment of mesenchymal stem cells from the local environment in the 3D scaffold (55).

Gene editing technology, such as the clustered regularly interspaced short palindromic repeat (CRISPR)/Cas9 system, improves the bacterial immune system and uses single guide RNA (sgRNA) to further activate the Cas9 endonuclease at the action site (56). This facilitates further DNA cleavage at the target site. Advanced technology, such as the CRISPR/ Cas9 system, employs genome engineering, the integration of which into 3DP applications may present a powerful gene therapy method (57). Gene-editing via a CRISPR/Cas system has been introduced to modify the genomes of various microorganisms and eukaryotic cells (58). The CRISPR/ Cas9 system can be used to reprogram primary fibroblasts to produce induced pluripotent stem cells (IPSCs). IPSCs themselves may differentiate into other cell types, such as mesenchymal stem cells and keratinocytes, representing the most significant cells for wound regeneration (59). Furthermore, the CRISPR/Cas system is used in gene therapy for wound healing and skin diseases. Here, the designed gene therapy system enhances the expression of skin growth factors, such as the epidermal growth factor, the platelet-derived growth factor, the transforming growth factor- $\beta$, and the fibroblast growth factor. In addition, the concept of using the gene-editing tool, CRISPR/Cas, to reprogram stem cells (such as IPSCs) was proposed, where the reprogrammed cells could be integrated into the made 
3D scaffold for wound repair applications. However, this system requires more research to realize its full potential (6).

A recent study has made progress regarding leukemia treatment. Leukemia stem cells (LSCs) refer to pathogenic acute myeloid leukemia (AML) cells and are among the reasons for AML recurrence. Treatments that remove LSCs may increase a patient's chances of overcoming this type of cancer (60). Therefore, a lipid-encapsulated Cas9/sgRNA ribonucleoprotein [lipid nanoparticles (LNP)-Cas9RNP] was used to target the key gene, interleukin-1 receptor helper in human LSC protein (IL1RAP). To enhance LSC targeting, the LNP-Cas9RNP and chemokine CXCL12 were loaded onto the nanofiber (MSCM-NF) scaffold wrapped in the membrane of the mesenchymal bone marrow stem cells that mimic the bone marrow microenvironment. The results showed that CXCL12RNP release caused LSC migration to the scaffold, while the LNP-Cas9RNP induced efficient gene editing. IL1RAP gene knockout reduced the colony-forming ability of LSC and the burden of leukemia, while stent administration increased the LNP-Cas9 residence time in the bone marrow cavity (61). In conclusion, the continuous local delivery of Cas9/IL1RAP sgRNA via the CXCL12LNP/NF scaffold provides an effective strategy for inhibiting LSC growth to improve AML treatment.

Although various clinical treatments for skin burn healing have shown reasonable results, more than $50 \%$ of wounds still fail to heal (62). Over the past 10 years, 3D bioprinting has made significant progress in regenerating transplantable tissues and even organs (such as skin, bones, and ears) to restore or repair damaged bodies. A recent study used human amniotic epithelial cells (AECs) and Wharton's jelly-derived mesenchymal stem cells (WJMSCs) as seeds. The double-layered membrane structures of the cells were obtained via 3D bioprinting. Human vascular endothelial cells showed better epithelial cell phenotypes during in vitro experiments, while WJMSCs exhibited enhanced angiogenic potential and fibroblast phenotypes. A higher cell survival rate (>95\%) was also observed 6 days after printing, showing potential for the future use of $3 \mathrm{D}$ bioprinting technology for skin engineering (63). The overall situation regarding the research involving gene therapy or the regulation using scaffolds obtained via $3 \mathrm{D}$ bioprinting in this article is depicted in Figure 2.

\section{Future trends}

\section{Gene-printing}

3D bioprinting provides a carrier and delivery system for seed cells. It is worth noting that the relationship between the structure of the molecule and its function is crucial. Developing dynamic 3D bioprinting models rely on their biochemical information, while the molecular information provided by nucleic acids, their interactions, functions, and specific mechanisms may lead to enhanced delivery systems (27). However, current DNA synthesis methods face several challenges, such as errors caused by genome sequencing, excessively long oligonucleotides, low yields, time-consuming processes, and the limitations presented by the laboratory environment and conditions (27). Therefore, direct gene-printing has attracted increasing attention since it provides a fast, convenient, and efficient way for producing nucleic acids while simultaneously avoiding the limitations mentioned above during the production process. San Francisco-based Cambrian Genomics has developed a DNA production technology based on laser printing. The method involves launching a computer-controlled laser beam into a glass tray containing millions of metal beads, each of which includes DNA. The impact of the laser pushes the beads carrying the correct DNA into the tray and screens them. Good DNA is sent to the collected flowing cells, leaving behind any unwanted or defective DNA. This process exponentially increases the DNA production process, making it faster, more convenient, and economical (64). It can be used to develop specific genome sequences for the gene therapy of human diseases, such as using genetic engineering to develop new drugs or to change protein sequences in tumor diseases.

Synthetic Genomics has developed a fully automated DNA printer (BioXp 3200 system) that first submits the gene sequence to the software, after which customized reagents are selected. The system is then loaded and activated to sequence and clone the DNA fragments. The pUCGA 1.0 DNA clone obtained from the BioXp system displayed a high cloning efficiency. The cloning efficiency of DNA fragments less than $900 \mathrm{bp}$ in length exceeded $90 \%$, while the overall cloning efficiency was $83 \%$ (65).

\section{D printing}

$4 \mathrm{D}$ printing may replace $3 \mathrm{D}$ printing as an effective platform for seed cell and gene delivery in the future. Traditional 3D printing only considers the initial state of the printed object and assumes it is an inanimate static scaffold. However, this insufficient for responding to the complex microenvironment in the body and constructing tissues or organs (66). 4D printing is defined as " $3 \mathrm{D}$ printing + time", in which the features (such as shape, 


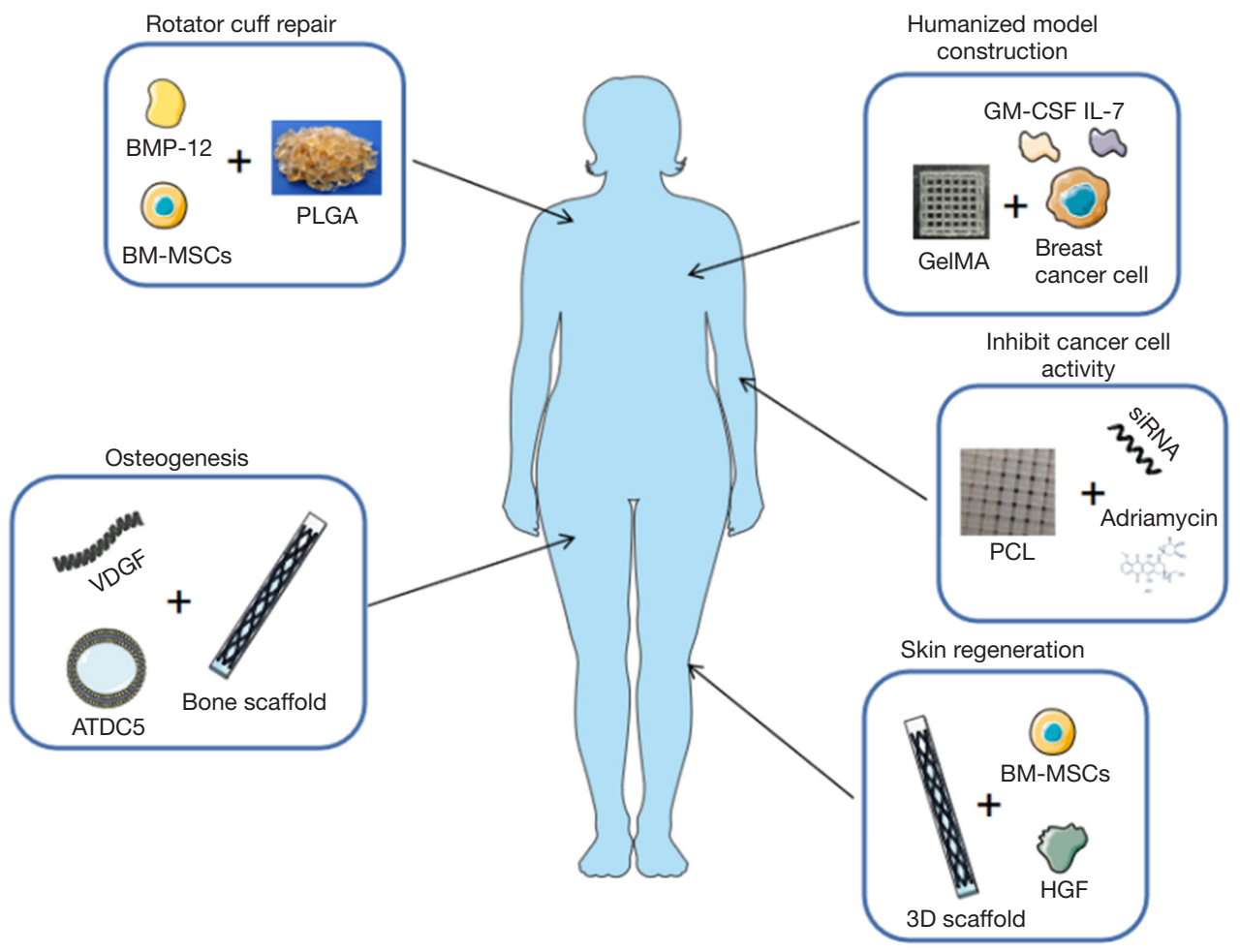

Figure 2 Overall summary of 3D printed scaffolds used in gene therapy. BMP-12, bone morphogenetic protein 12; BM-MSCs, bone marrow-derived mesenchymal stem cells; GeIMA, gelatin methacryloyl; GM-CSF, granulocyte-macrophage colony-stimulating factor; HGF, hepatocyte growth factor; IL-7, interleukin-7; PCL, poly(E-caprolactone); VEGF, vascular endothelial growth factor.

attributes, or functions) of the manufactured object can be stimulated via internal and external environmental stimuli (such as chemical agents, temperature, mechanical stress, radiation, and $\mathrm{pH}$, as well as electrical and magnetic fields), which changes over time $(67,68)$. This technique may be useful in tissue engineering.

However, 4D printing currently faces many obstacles, which it must overcome to fully realize its potential in manufacturing technology. Some of the main obstacles include a decrease in the mechanical strength of the material and a longer response time to stimuli, resulting in a slower shape change rate (69). Therefore, more energy and expertise should be invested in developing new multifunctional 4D inks to improve 4D printing technology.

\section{Conclusions}

3D bioprinting combined with gene-editing technology provides an efficient and accurate delivery system for personalized tumor therapy, enhancing the targeting effect while maintaining the integrity of the fabricated structure. It exhibits significant application potential in developing tumor drugs. In addition, scaffolds obtained via $3 \mathrm{D}$ bioprinting provide gene therapy applications for skin and bone healing and repair and inducing stem cell differentiation. However, the research in this field has remained preliminary or at the laboratory stage for many years. This may be attributed to the complexity of the cells and microenvironment in the human body and failure to establish good interaction between the materials and the cells, making it challenging to maintain or simulate the in vivo environment. This has widened the gap between laboratory research and clinical application in this field. In addition, the cells and genes used for 3D bioprinting must be regulated to address product functionality and ethical challenges. However, with the further clinical development and 4D printing technology, combined with the maturity of gene-editing technology, this field exhibits considerable potential for research and disease treatment in the future. 


\section{Acknowledgments}

Funding: This work was supported by the National Natural Science Foundation of China (grant number 31972915) to WH; the Science and Technology Project of Guangdong Province (grant number 2016B090917001) to WH; Sanming Project of Medicine in Shenzhen (grant number SZSM201612019) to WH; Sichuan Applied Basic Research Project (grant number 2018JY0402) to HL; Luzhou Municipal People's Government-Southwest Medical University Science and Technology Strategic Cooperation Project (grant number 2018LZXNYD-ZK19) to HL.

\section{Footnote}

Reporting Checklist: The authors have completed the Narrative Review reporting checklist. Available at https:// dx.doi.org/10.21037/atm-21-2854

Peer Review File: Available at https://dx.doi.org/10.21037/ atm-21-2854

Conflicts of Interest: All authors have completed the ICMJE uniform disclosure form (available at https://dx.doi. org/10.21037/atm-21-2854). The authors have no conflicts of interest to declare.

Ethical Statement: The authors are accountable for all aspects of the work in ensuring that questions related to the accuracy or integrity of any part of the work are appropriately investigated and resolved.

Open Access Statement: This is an Open Access article distributed in accordance with the Creative Commons Attribution-NonCommercial-NoDerivs 4.0 International License (CC BY-NC-ND 4.0), which permits the noncommercial replication and distribution of the article with the strict proviso that no changes or edits are made and the original work is properly cited (including links to both the formal publication through the relevant DOI and the license). See: https://creativecommons.org/licenses/by-nc-nd/4.0/.

\section{References}

1. Li X, Ma J, L P, et al. 3D printing technology and its application trend. Process Automation Instrumentation 2014;35:1-5.

2. Liu W, Li Y, Liu J, et al. Application and Performance of 3D Printing in Nanobiomaterials. Journal of Nanomaterials 2013;2013:681050.

3. Gu Z, Fu J, Lin H, et al. Development of 3D bioprinting: From printing methods to biomedical applications. Asian J Pharm Sci 2020;15:529-57.

4. Malda J, Visser J, Melchels FP, et al. 25th anniversary article: Engineering hydrogels for biofabrication. Adv Mater 2013;25:5011-28.

5. Kamisuki S, Hagata T, Tezuka C, et al. A low power, small, electrostatically-driven commercial inkjet head. Proceedings MEMS 98. IEEE. Eleventh Annual International Workshop on Micro Electro Mechanical Systems. An Investigation of Micro Structures, Sensors, Actuators, Machines and Systems (Cat. No.98CH36176). 25-29 Jan. 1998; Heidelberg, Germany. IEEE, 1998:63-8.

6. Sears NA, Seshadri DR, Dhavalikar PS, et al. A Review of Three-Dimensional Printing in Tissue Engineering. Tissue Eng Part B Rev 2016;22:298-310.

7. Masood SH. Intelligent rapid prototyping with fused deposition modelling. Rapid Prototyping Journal 1996;2:24-33.

8. Wijshoff $\mathrm{H}$. The dynamics of the piezo inkjet printhead operation. Physics Reports 2010;491:77-177.

9. Gudapati H, Dey M, Ozbolat I. A comprehensive review on droplet-based bioprinting: Past, present and future. Biomaterials 2016;102:20-42.

10. Gurkan UA, El Assal R, Yildiz SE, et al. Engineering anisotropic biomimetic fibrocartilage microenvironment by bioprinting mesenchymal stem cells in nanoliter gel droplets. Mol Pharm 2014;11:2151-9.

11. Guillotin B, Guillemot F. Cell patterning technologies for organotypic tissue fabrication. Trends Biotechnol 2011;29:183-90.

12. Hinton TJ, Jallerat Q, Palchesko RN, et al. Threedimensional printing of complex biological structures by freeform reversible embedding of suspended hydrogels. Sci Adv 2015;1:e1500758.

13. Nelson CE, Duvall CL, Prokop A, et al. Gene delivery into cells and tissues. In: Lanza R, Langer R, Vacanti J, et al. editors. Principles of Tissue Engineering. 5th edition. American Academic Press, 2020:519-54.

14. Cevher E, Sezer AD, Çaglar ES, Gene Delivery Systems: Recent Progress in Viral and Non-Viral Therapy. In: Sezer AD. Recent Advances in Novel Drug Carrier Systems. IntechOpen, 2012:437-70.

15. Mofazzal Jahromi MA, Sahandi Zangabad P, Moosavi Basri SM, et al. Nanomedicine and advanced technologies for burns: Preventing infection and facilitating wound healing. 
Adv Drug Deliv Rev 2018;123:33-64.

16. Goswami R, Subramanian G, Silayeva L, et al. Gene Therapy Leaves a Vicious Cycle. Front Oncol 2019;9:297.

17. Gutierrez L, Cauchon NS, Christian TR, et al. The Confluence of Innovation in Therapeutics and Regulation: Recent CMC Considerations. J Pharm Sci 2020;109:3524-34.

18. Beck RCR, Chaves PS, Goyanes A, et al. 3D printed tablets loaded with polymeric nanocapsules: An innovative approach to produce customized drug delivery systems. Int J Pharm 2017;528:268-79.

19. Alluri R, Jakus A, Bougioukli S, et al. 3D printed hyperelastic "bone" scaffolds and regional gene therapy: A novel approach to bone healing. J Biomed Mater Res A 2018;106:1104-10.

20. Ashwin B, Abinaya B, Prasith TP, et al. 3D-poly (lactic acid) scaffolds coated with gelatin and mucic acid for bone tissue engineering. Int J Biol Macromol 2020;162:523-32.

21. Martin V, Ribeiro IA, Alves MM, et al. Engineering a multifunctional 3D-printed PLA-collagen-minocyclinenanoHydroxyapatite scaffold with combined antimicrobial and osteogenic effects for bone regeneration. Mater Sci Eng C Mater Biol Appl 2019;101:15-26.

22. Lewicki J, Bergman J, Kerins C, et al. Optimization of 3D bioprinting of human neuroblastoma cells using sodium alginate hydrogel. Bioprinting 2019;16:e0053.

23. Xin $X$, Jie T, Shuai $W$, et al. 3D printing of nerve conduits with nanoparticle-encapsulated RGFP966. Applied Materials Today 2019;16:247-56.

24. Nishiguchi A, Shima F, Singh S, et al. 3D-Printing of Structure-Controlled Antigen Nanoparticles for Vaccine Delivery. Biomacromolecules 2020;21:2043-8.

25. Chan K, Wong PY, Parikh C, et al. Moving toward rapid and low-cost point-of-care molecular diagnostics with a repurposed $3 \mathrm{D}$ printer and RPA. Anal Biochem 2018;545:4-12.

26. Tack P, Victor J, Gemmel P, et al. 3D-printing techniques in a medical setting: a systematic literature review. Biomed Eng Online 2016;15:115.

27. Shende P, Trivedi R. 3D Printed Bioconstructs: Regenerative Modulation for Genetic Expression. Stem Cell Rev Rep 2021;17:1239-50.

28. Saleh Alghamdi S, John S, Roy Choudhury N, et al. Additive Manufacturing of Polymer Materials: Progress, Promise and Challenges. Polymers (Basel) 2021;13:753.

29. Shende P, Patil A, Prabhakar B. Layer-by-layer technique for enhancing physicochemical properties of actives. J Drug Deliv Sci Technol 2020;56:101519.

30. Gallo RC, Whang-Peng J, Adamson RH. Studies on the antitumor activity, mechanism of action, and cell cycle effects of camptothecin. J Natl Cancer Inst 1971;46:789-95.

31. Jones CB, Clements MK, Wasi S, et al. Sensitivity to camptothecin of human breast carcinoma and normal endothelial cells. Cancer Chemother Pharmacol 1997;40:475-83.

32. Landgraf M, Lahr CA, Kaur I, et al. Targeted camptothecin delivery via silicon nanoparticles reduces breast cancer metastasis. Biomaterials 2020;240:119791.

33. Moreau JE, Anderson K, Mauney JR, et al. Tissueengineered bone serves as a target for metastasis of human breast cancer in a mouse model. Cancer Res 2007;67:10304-8.

34. Bersani F, Lee J, Yu M, et al. Bioengineered implantable scaffolds as a tool to study stromal-derived factors in metastatic cancer models. Cancer Res 2014;74:7229-38.

35. Escobar G, Moi D, Ranghetti A, et al. Genetic engineering of hematopoiesis for targeted IFN- $\alpha$ delivery inhibits breast cancer progression. Sci Transl Med 2014;6:217ra3.

36. Shafiee A, McGovern JA, Lahr CA, et al. Immune system augmentation via humanization using stem/progenitor cells and bioengineering in a breast cancer model study. Int J Cancer 2018;143:1470-82.

37. Morrot A, da Fonseca LM, Salustiano EJ, et al. Metabolic Symbiosis and Immunomodulation: How Tumor CellDerived Lactate May Disturb Innate and Adaptive Immune Responses. Front Oncol 2018;8:81.

38. Allen R, Ivtchenko E, Thuamsang B, et al. Polymer-loaded hydrogels serve as depots for lactate and mimic "cold" tumor microenvironments. Biomater Sci 2020;8:6056-68.

39. Holzapfel BM, Wagner F, Thibaudeau L, et al. Concise review: humanized models of tumor immunology in the 21st century: convergence of cancer research and tissue engineering. Stem Cells 2015;33:1696-704.

40. Theocharides AP, Rongvaux A, Fritsch K, et al. Humanized hemato-lymphoid system mice. Haematologica 2016;101:5-19.

41. Wagner F, Holzapfel BM, McGovern JA, et al. Humanization of bone and bone marrow in an orthotopic site reveals new potential therapeutic targets in osteosarcoma. Biomaterials 2018;171:230-46.

42. Cloyd JM, Acosta FL Jr, Polley MY, et al. En bloc resection for primary and metastatic tumors of the spine: a systematic review of the literature. Neurosurgery 2010;67:435-44; discussion 444-5.

43. Yohe ST, Herrera VL, Colson YL, et al. 3D superhydrophobic electrospun meshes as reinforcement materials for sustained local drug delivery against 
colorectal cancer cells. J Control Release 2012;162:92-101.

44. Chen M, Andersen MØ, Dillschneider P, et al. Co-delivery of siRNA and doxorubicin to cancer cells from additively manufactured implants†. RSC Adv 2015;5:101718-25.

45. Li S, Sun H, Luo X, et al. The clinical effect of rehabilitation following arthroscopic rotator cuff repair: A meta-analysis of early versus delayed passive motion. Medicine (Baltimore) 2018;97:e9625.

46. Louati H, Uhthoff HK, Culliton K, et al. Supraspinatus tendon repair using anchors: a biomechanical evaluation in the rabbit. J Orthop Surg Res 2018;13:64.

47. Ellera Gomes JL, da Silva RC, Silla LM, et al.

Conventional rotator cuff repair complemented by the aid of mononuclear autologous stem cells. Knee Surg Sports Traumatol Arthrosc 2012;20:373-7.

48. Heisterbach PE, Todorov A, Flückiger R, et al. Effect of BMP-12, TGF- $\beta 1$ and autologous conditioned serum on growth factor expression in Achilles tendon healing. Knee Surg Sports Traumatol Arthrosc 2012;20:1907-14.

49. Chen P, Cui L, Chen G, et al. The application of BMP-12overexpressing mesenchymal stem cells loaded 3D-printed PLGA scaffolds in rabbit rotator cuff repair. Int J Biol Macromol 2019;138:79-88.

50. Abu Awwad HAM, Thiagarajan L, Kanczler JM, et al. Genetically-programmed, mesenchymal stromal cell-laden \& mechanically strong 3D bioprinted scaffolds for bone repair. J Control Release 2020;325:335-46.

51. Daley GQ, Scadden DT. Prospects for stem cell-based therapy. Cell 2008;132:544-8.

52. Zha Y, Li Y, Lin T, et al. Progenitor cell-derived exosomes endowed with VEGF plasmids enhance osteogenic induction and vascular remodeling in large segmental bone defects. Theranostics 2021;11:397-409.

53. Church D, Elsayed S, Reid O, et al. Burn wound infections. Clin Microbiol Rev 2006;19:403-34.

54. Peck M, Molnar J, Swart D. A global plan for burn prevention and care. Bull World Health Organ 2009;87:802-3.

55. van de Kamp J, Paefgen V, Wöltje M, et al. Mesenchymal stem cells can be recruited to wounded tissue via hepatocyte growth factor-loaded biomaterials. J Tissue Eng Regen Med 2017;11:2988-98.

Cite this article as: Fu B, Shen J, Chen Y, Wu Y, Zhang H, Liu H, Huang W. Narrative review of gene modification: applications in three-dimensional (3D) bioprinting. Ann Transl Med 2021;9(19):1502. doi: 10.21037/atm-21-2854
56. Martinez-Lage M, Torres-Ruiz R, Rodriguez-Perales S. CRISPR/Cas9 Technology: Applications and Human Disease Modeling. Prog Mol Biol Transl Sci 2017;152:23-48.

57. Gopal S, Rodrigues AL, Dordick JS. Exploiting CRISPR Cas9 in Three-Dimensional Stem Cell Cultures to Model Disease. Front Bioeng Biotechnol 2020;8:692.

58. Sander JD, Joung JK. CRISPR-Cas systems for editing, regulating and targeting genomes. Nat Biotechnol 2014;32:347-55.

59. Chen M, Przyborowski M, Berthiaume F. Stem cells for skin tissue engineering and wound healing. Crit Rev Biomed Eng 2009;37:399-421.

60. Ding L, Ley TJ, Larson DE, et al. Clonal evolution in relapsed acute myeloid leukaemia revealed by wholegenome sequencing. Nature 2012;481:506-10.

61. Ho TC, Kim HS, Chen Y, et al. Scaffold-mediated CRISPR-Cas9 delivery system for acute myeloid leukemia therapy. Sci Adv 2021;7:eabg3217.

62. Hirt-Burri N, Ramelet AA, Raffoul W, et al. Biologicals and fetal cell therapy for wound and scar management. ISRN Dermatol 2011;2011:549870.

63. Liu $\mathrm{P}$, Shen $\mathrm{H}, \mathrm{Zhi} \mathrm{Y}$, et al. 3D bioprinting and in vitro study of bilayered membranous construct with human cells-laden alginate/gelatin composite hydrogels. Colloids Surf B Biointerfaces 2019;181:1026-34.

64. DNA 'Printing' A Big Boon To Research, But Some Raise Concerns [Internet].2021 January 5, Available online: https://3 dprintingindustry.com/news/cambrian-genomics3d-printing-dna-25831/

65. Alvarez CH. DNA Assembly and Cloning in an Overnight Run with the BioXp ${ }^{\text {TM }} 3200$ System. Nature Methods 2015;12:v-vi.

66. Yang Q, Gao B, Xu F. Recent Advances in 4D Bioprinting. Biotechnol J 2020;15:e1900086.

67. Momeni F, Hassani SMM, Liu X, et al. A review of 4D printing. Materials \& Design 2017;122:42-79.

68. Kaynak A, Zolfagharian A. Stimuli-Responsive Polymer Systems-Recent Manufacturing Techniques and Applications. Materials (Basel) 2019;12:2380.

69. Bajpai A, Baigent A, Raghav S, et al. 4D Printing: Materials, Technologies, and Future Applications in the Biomedical Field. Sustainability 2020;12:10682. 